\title{
Application and Characterization Osteo Nanoporation under Electrical Stimuli
}

\section{Sarkar $\mathbf{S}^{1 *}$ and Ghose $\mathbf{M K}^{2}$}

${ }^{1}$ Department of ECE, SMIT, Sikkim, India

${ }^{2}$ Dean Academic, SMIT, India

\begin{abstract}
Osteo intraorganelle nanoporation is a highly effective method to increase permeability of intraorganelle membrane of rigid bone cell under the influences of pico electric pulses and using this technique, we can introduce specific drugs into the nucleus of the rigid cell like osteoblast. It is also a promising technology in drug delivery system and localization of malignant cancer cell. In this research paper the characterization and usefulness of osteo nanoporation is described. In this context specific nanoporative device simulation and its effect on intra organelle nanoporation are explored and at the end different application of nanoporation are depicted.
\end{abstract}

Keywords: Pico seconds pulsed electric field (psPEF); Bi-metallic electrode; Integrated bio microchip; Osteoblast cell; Intra-organelle; Nanoporation; Membrane pore; Ion uptake; Drug delivery system

\section{Introduction}

The role of short and intense electric fields on biological cells have been studied for a long time as they are known to apply interrupt on the plasma membrane which can become permeable to various molecules [1]. This temporary permeabilization of the plasma membrane allows genes or drugs entering into the cell intraorganelle. This technique is very attractive in various applications such as electro-chemotherapy $[2,3]$, cutaneous and subcutaneous tumour nodule treatments $[4,5]$, evaluation of the cytotoxici of non-permanent or poorly permanent anticancer drugs [6], gene electro transfer to various animal tissues [7] and latterly with in various tests [8]. Permeabilization of the cell plasma membrane with high-amplitude electric pulses has been known for decades and is commonly employed for intracellular delivery of xenobiotic and cell destruction $[9,10]$. Shortening the pulse duration into nanosecond range has the potential to permeabilize not only the plasma membrane, but the intracellular membranous structures as well $[11,12]$. Multiple studies have attempted to verify this theoretical prediction, with rather diverse outcomes. Depending on the experimental approach, the studies reported only plasma membrane effects $[13,14]$. Different works have reported about the effects on animal cells of these kinds of pulses, commonly named nanosecond pulsed electric fields (nsPEF). However, the mechanisms implied in the effects of psPEF on living cells remain still misunderstood $[15,16]$. This is mainly due to the lack of real-time visualization and monitoring systems during psPEF application on biological cells $[17,18]$.

The use of micro fluidic devices in nanoporation is a remarkable exploration in the field of drug delivery system [19]. Although the nanoporation process itself in sophisticated devices with full control over the process as well as cell debris separation and subsequent intracellular cell content analysis have not been reported yet [20]. Therefore, it is expected that integrated devices where combinations of electroporation, separation and analysis occur will emerge, such as devices with integrated chromatography [20], electrophoresis [21] or iso-electric focusing steps for separation, and mass spectroscopic, electrochemical and fluorescent methods for analysis [22]. Secondly, the present designs usually require multiple manual steps in order to insert the cells, electroporation them and measure the effects. Until now, no devices have been reported where all of these steps have been integrated in an automated way, preferably with multiple samples in 482 parallel, which could greatly enhance the application of micro technological analysis [23-27]. Author also proposed integrated devices where combinations of nanoporation, separation and analysis occur will occur, and all of these steps have been integrated in an externally regulated automated way. As the cells are in contact together tightly so in this thesis the neighbour cells are also considered to make the result more important and realistic.

In the other hand Electric stimulation has been used successfully to treat a wide range of bone disorders. However, the mechanism by which the electric fields can influence the bone cells behaviour remains poorly understood [28]. The purpose of this research was to assess the possible mechanism of the stimulatory effect of Pulsed Electromagnetic Field (PEMF) and intraorganelle pore formation on osteoblast like bone cells. Therefore further research is required to determine this interaction process between electric fields and bone cells. This was exactly the objective that motivated the present work "to identify the possible pathway(s) of interaction between electric fields and bone cells". However, despite an abundance of undeniably valid data, from both basic and multicenter clinical trials, demonstrating the effects of electrical stimulation on skeletal biology and healing, little is known about the signal transduction that mediates the physiological response of bone to electrical stimulation [29].

As electrical stimulation have been used electric and magnetic fields, direct current and ultrasound among others. Bone cells have responded in different manners that are considered key in the process of bone formation. Therefore it has not been questionable if bone cells respond to electrical stimulation, but what is the mechanism that is acting on the cells, and how it is transduced into biochemical signals for generating an appropriate physiological response [30]. In light of all research done with bone and electricity, there is enough evidence to say that electric fields exist into bone and play a role in bone physiology, and that external electrical stimulation can also alter profoundly many cellular events and modulate the bone physiology but the mechanism for pore formation is not properly investigate. However, until the advance mechanism of interaction between electric fields and bone

*Corresponding author: Sarkar S, Department of ECE, SMIT, Sikkim, India, Tel: 3592-246466; E-mail: swaupsarkar957@yahoo.com

Received April 29, 2015; Accepted May 30, 2015; Published June 03, 2015

Citation: Sarkar S, Ghose MK (2015) Application and Characterization Osteo Nanoporation under Electrical Stimuli. Gene Technology 4: 123. doi: 10.4172/23296682.1000123

Copyright: (C) 2015 Sarkar S, et al. This is an open-access article distributed under the terms of the Creative Commons Attribution License, which permits unrestricted use, distribution, and reproduction in any medium, provided the original author and source are credited. 
cells placed in a microfluidics is limited, little advance can be made in the intraorganelle pore formation into the osteoblast like bone cell forthe management of bone diseases with electrotherapy [31].

The micro electroporation chip was designed and fabricated using standard silicon micro fabrication technology. With the ability to manipulate individual cells, the chip can be used to study the fundamental biophysics of intraorganelle nanoporation of single osteoblast like-cell level and in biotechnology, for controlled introduction of macromolecules, such as gene constructs, into individual cells. The general principle of the microelectroporation technology and the design, fabrication of chip and the characterization of nanoporations are presented after the first description of in vitro gene transfer to living cells by intraorganelle nanoporation (EP) this technique has been widely used. The use of intraorganelle nanoporation to transport non-permeant chemotherapy drugs has been termed electrochemotherapy.

Nanoporation-based technologies and medical applications have already shown their laboratory and clinical relevance. It is becoming a standard appliances in bi-medical applications are in progress. Although the number of successful applications is increasing, several questions concerning the optimization of pulse parameters for specific application are still open. Among them is determination of appropriate amplitude, duration, number, and repetition frequency of electric pulses that assure successful application or treatment with minimal possible side effects.

In this paper major focus will be on numerical analysis of nanoporation and simulation model of 3D micro device used for intraorganelle characterization for osteoblast cell and various biomedical applications of intraorganelle nanoporation under the influences of specific micro bio device.

\section{Numerical Models}

\section{Analytical modelling of nanoporation}

The Smoluchowski equation defines a pore density function, $n(r, t)$, such that the number of pores with radius between $r$ and $r+d r$ at any given time, $t$, is $n(r, t) d r . n(r, t)$ is described as

$$
\frac{\partial n}{\partial t}+D \frac{\partial}{\partial r}\left(-\frac{\partial n}{\partial r}-\frac{n}{k T} \frac{\partial W}{\partial r}\right)=S(r)
$$

Where $D$ is the pore diffusion coefficient, $r$ is the pore radius, $W$ is the formation energy of a pore with radius $r$, and $S(r)$ describes the transition of hydrophobic pores to hydrophilic ones, as

$$
S(r)=\frac{v_{c}}{k T} h \frac{\partial W_{0}}{\partial r} e^{W_{0} / k T}-v_{d} n H\left(r_{*}-r\right)
$$

where $v c$ is the pore creation rate, $h$ is the membrane thickness, Wo is the formation energy of a hydrophobic pore, $v d$ is the pore destruction rate, $r *$ is the radius at which hydrophobic and hydrophilic pores have the same energy, and $H(r *-r)$ is a step function at $r=r *$. Assuming that, (i) the expansion of the pores is negligible, and (ii) the temporal change of the minimum pore energy is negligible, a quasistatic asymptotic model of ntraorganelle nanoporation simplifies the PDE equation to I an ordinary differential equation. The ODE defines the pore density, $N(t)$, which is related to $n(r, t)$ as

$$
N(t)=\int_{r=0}^{\infty} n(r, t) d r
$$

The quasistatic asymptotic equation for $N(t)$ is

$$
\frac{d N(t)}{d t}=\propto e^{\left(V_{m}(t) / V_{e p}\right)^{2}}\left(1-\frac{N(t)}{N_{\mathrm{O}}} e^{-q\left(V_{m}(t) / V_{e p}\right)^{2}}\right)
$$

Where $V m$ is the transmembrane voltage, Vep is the characteristic voltage of Intraorganelle nanoporation, $N 0$ is the equilibrium pore density at $V m=0$, and $a$ and $q$ are constants.

It is reported that, when the transmembrane voltage achieves the required voltage of Intraorganelle nanoporation some pores are formed in the membrane. The formation of the pores increases the membrane conductivity of the membrane and is electrically modelled as an additional current density, Jep, inside the membrane. Jep is written as

$$
J_{e p}(t)=N(t) \frac{\pi r_{p}^{2}}{h} \sigma_{p} V_{m} K
$$

where $N$ is the density of the pores $r p$ is the pore radius, $\sigma p$ is the conductivity of the solution inside the pore, $\mathrm{Vm}$ is the transmembrane voltage, $h$ is the thickness of the membrane, and $K$ is

$$
K=\frac{e^{v_{m}}-1}{\frac{\omega_{0} e^{\omega_{0}-n v_{m}}-n v_{m}}{\omega_{0}-n v_{m}} e_{m}^{v}-\frac{\omega_{0} e^{\omega_{0}+n v_{m}}+n v_{m}}{\omega_{0}+n v_{m}}}
$$

Where $W 0$ is the energy barrier inside the pore, $n$ is the relative entrance length of the pore, and $v m=q e, K T V$,is the non-dimensional transmembrane voltage. Assuming that the electric field inside the membrane is uniform, the transmembrane voltage is written as

$V_{m}=E_{\star} h$

Where $E_{\star}$ is normal electric field.

The pore current density inside the membrane can be translated as an increase in the membrane conductivity using the relation

$$
J_{c p}=\sigma_{m} E_{*}
$$

The conductivity of the membrane at the points that pores are formed is calculated as

$$
\sigma_{m}(t)=\sigma_{m} 0+N(t) \sigma_{p} \sigma \sigma r_{p} \sigma^{2} K
$$

where $\sigma m 0$ is the conductivity of the membrane before Intraorganelle nanoporation. The nonlinearity of the equation 3.4 comes from $N$ which was described as

$$
\frac{d N(t)}{d t}=\propto e^{\left(V_{m}(t) / V_{e p}\right)^{2}}\left(1-\frac{N(t)}{N_{\mathrm{O}}} e^{-q\left(V_{m}(t) / V_{e p}\right)^{2}}\right)
$$

Under equilibrium conditions (9) and (10) maintain the transmembrane voltage below the required voltage of Intraorganelle nanoporation, which is about $1 \mathrm{~V}$. Required voltage of nanoporation is the threshold at which notable increase in the density of the pores and membrane conductivity and consequently decrease in the transmembrane voltage occur. The increase in the transmembrane voltage leads to the increase of pore density which lowers the conductivity of the membrane and subsequently the transmembrane voltage.

\section{Simulation model bio chip}

Design of bio chip: It is reported that the effective nanoporation in intraorganelle membrane or nucleolus membrane need specific type of pulse, microfloudic chip and suspension media. It is reported that the optimum value of the applied pulse having the duration of 5 pico second, pulse intensity of 1 volts is suitable and effective intraorganelle nanoporation of osteoblast cell. It also exposed that intraorganelle nanoporation can easily obtained if the shape of the electrode is triangular or saw tooth which are made by gold bismuth alloy in specified micro channel having the height and width of micro-fluidic channel should be moderate and same and it would be taken as 200 micrometer for successful and effective intraorganelle nanoporation. The resistances of micro channel should be as high as possible for above purpose. With the help of above discussion the author optimised 
the system and design a simulating micro fluidic chip on the bases of following specification with in which the osteoblast cell is placed, to explore the numerical and analytical characterization of intraorganelle nanoporation. The simulation tool is composed of a $100 \mu \mathrm{m}$ thick SU8 micro fluidic channel including thick bi metallic electrode (Bi and $\mathrm{Au}$ ) electrodes with a typical thickness of $50 \mu \mathrm{m}$, in which cells suspended in a biological medium are injected. Bi-metallic is chosen as material for the electrodes because of its excellent electrical properties and bio compatibility. The biochip is designed in such a way that the pulsed electric field is absorbed and dissipated mainly in the biological medium placed between the electrodes within which cells to be treated are flowed (Table 1).

Characterization of Micro chip: The biochip is validated by its frequency and time domain analysis. The electromagnetic property is measure by LCR meter along with dielectric probe and relative permittivity and reflection co efficient are measured by vector network analyser. The impedance is calculated from the reflection coefficient. Figure 1 explores the real part of impedances of bio chip. The measured results present a very good level of consistency. The good impedance matching is obtain up to $100 \mathrm{MHz}$. As shown in Figure 2 at low frequency the biochip is purely resistive but in high frequency the

\begin{tabular}{|c|c|c|c|}
\hline Devices & Parameter & Value & Unit \\
\hline \multirow{5}{*}{ Chip } & Length & 2300 & $\mu \mathrm{m}$ \\
\hline & Height & 100 & $\mu \mathrm{m}$ \\
\hline & Width & 900 & $\mu \mathrm{m}$ \\
\hline & Inlet & 10 & $\mu \mathrm{m}$ \\
\hline & Outlet & 10 & $\mu \mathrm{m}$ \\
\hline \multirow{4}{*}{ Micro } & Length & 1000 & $\mu \mathrm{m}$ \\
\hline & Width & 900 & $\mu \mathrm{m}$ \\
\hline & Height & 100 & $\mu \mathrm{m}$ \\
\hline & Inter electrode gap & & \\
\hline \multirow{4}{*}{ Electrode } & Central part & 50 & $\mu \mathrm{m}$ \\
\hline & Medial part & 150 & $\mu \mathrm{m}$ \\
\hline & Lateral part & 250 & $\mu \mathrm{m}$ \\
\hline & Material & $\mathrm{Au}, \mathrm{Bi}$ & $\mu \mathrm{m}$ \\
\hline \multirow{3}{*}{$\begin{array}{l}\text { Micro channel(Bi- } \\
\text { lateral) }\end{array}$} & Length & 2300 & $\mu \mathrm{m}$ \\
\hline & Width & 250 & $\mu \mathrm{m}$ \\
\hline & Height & 100 & $\mu \mathrm{m}$ \\
\hline
\end{tabular}

Table 1: Design consideration of the microchip in COMSOL software [33].

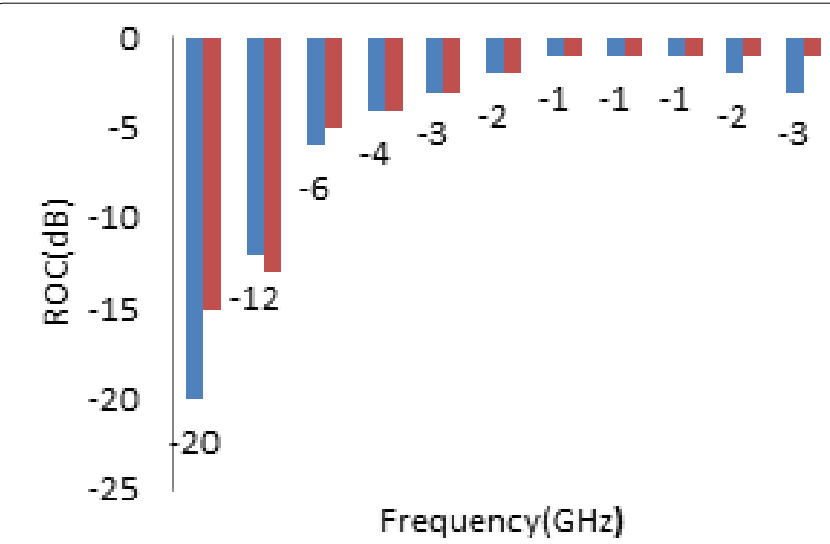

Figure 1: Frequency domain characterization of biochip with measurements of reflection coefficient.

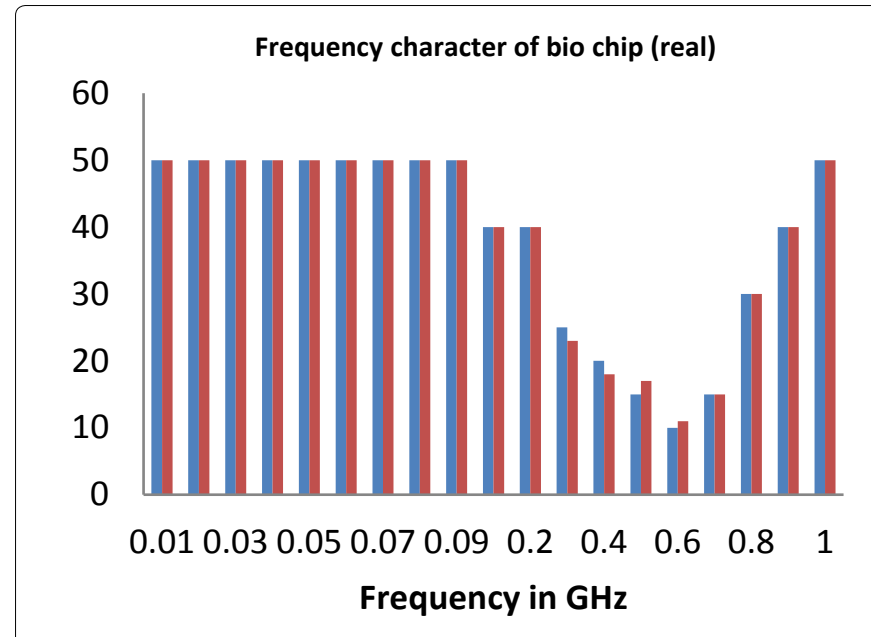

Figure 2: Frequency domain characterization of biochip, measurements of impedances.

Rice time 0.5 ps

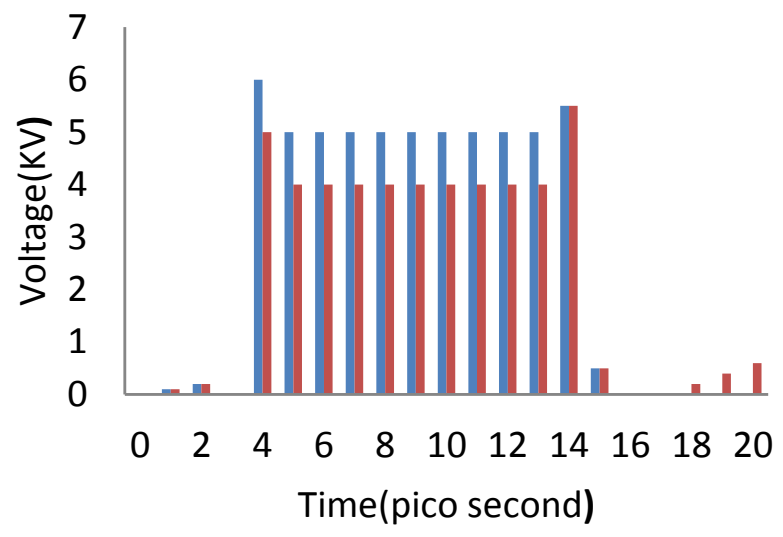

Figure 3: Time domain characterization of biochip, Measurements of voltage applied and generated within the bio chip. (Rice time $0.5 \mathrm{ps}$ ).

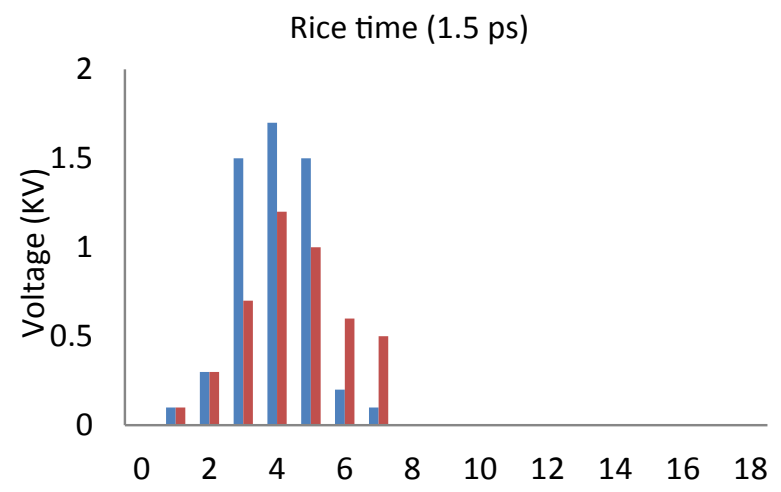

Figure 4: Time domain characterization of biochip, Measurements of voltage applied and generated within the bio chip. (Rice time $-1.5 \mathrm{ps}$ )

impedance of bio chip is reduced. In Figures 3 and 4 the voltage is measured, i.e. generated and applied pulses are present and it explores the good level of consistency. The rice time is slightly modified by the biochip and make it reliable for intraorganelle nanoporation. 


\section{Biological Experiments}

\section{Experimental process}

In this study the experimental biological tests are performed with 20 ps duration pulses. A train of 200 psPEF, square shaped, 20 ps duration is applied with a repetition frequency of $234 \mathrm{~Hz}$. A view (fluorescence or bright field microscopy) of cells within the 3D hybrid microchip is recorded before and just after the application of psPEF. As shown in Figure 5, Cells fluoresce in red after the application of psPEF, proving that PI introduces into the cytosol, due to the disturbance of the plasma membrane. Viability of cells submitted to psPEF treatment was checked by trypan Blue test. An average of $90 \pm 1 \%$ of exposed cells remains still alive $30 \mathrm{~min}$ after being exposed to ps PEF. The number of nano pulses that were applied (200 or 300 pulses), to confirm the effect of these parameters on the intra cellular nanoporation. As expected, the number of pulses and their amplitude both influence the level of permeabilization. These preliminary characterizations of the effect of

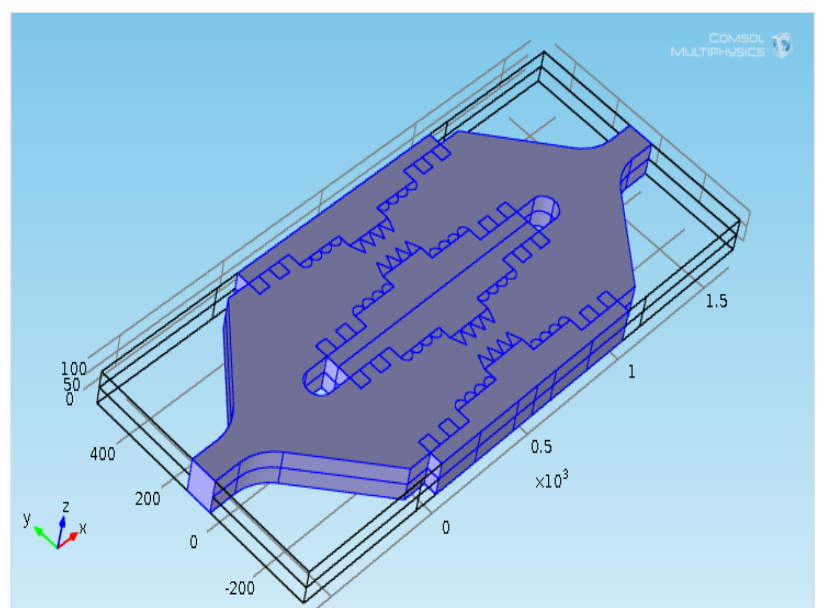

Figure 5: Design view of 3D micro device.

\begin{tabular}{|c|c|c|}
\hline \multicolumn{2}{|c|}{ Condition } & \% of permeabilizations \\
\hline $\begin{array}{c}\text { Applied electric field } \\
\text { (KV/Cm) }\end{array}$ & No of pulse & 58 \\
\hline 35 & 150 & 60 \\
\hline 40 & 175 & 72 \\
\hline 45 & 200 & 85 \\
\hline 50 & 200 & 87 \\
\hline 55 & 250 & 90 \\
\hline 60 & 250 & 95 \\
\hline 65 & 300 & \\
\hline
\end{tabular}

Table 2: Percentage of permeabilized cells.

\begin{tabular}{|c|c|}
\hline $\begin{array}{c}\text { Pulse duration } \\
\text { (X-axis) second }\end{array}$ & $\begin{array}{c}\text { Flu recent intensity (Y-axis)no of } \\
\text { molecules }\end{array}$ \\
\hline $10^{-6}$ & 0 \\
\hline $10^{-7}$ & 11,000 \\
\hline $10^{-8}$ & 16,000 \\
\hline $10^{-9}$ & 21,000 \\
\hline $10^{-10}$ & 41,000 \\
\hline $10^{-11}$ & 81,000 \\
\hline $10^{-12}$ & 101,000 \\
\hline $10^{-13}$ & 101,000 \\
\hline $10^{-14}$ & 101,000 \\
\hline & \\
\hline
\end{tabular}

Table 3: Experimental data.

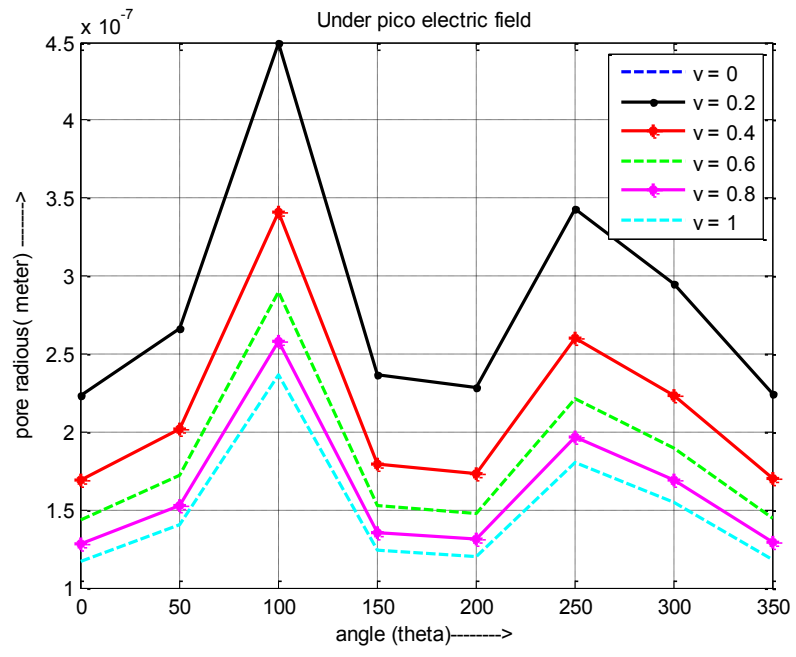

Figure 6: Variation of intraorganelle pore radius with respect to azimuthal angle in different pulse intensity.

nanoporation on intra organelle, in particular on the permeabilization of the plasma membrane, were permitted thanks to the real time Observation on the miniaturized bio device. Deeper exploitation of this type of device will be conducted together with the development of new psPEF generators. Actual results demonstrate the capability of the developed $3 \mathrm{D}$ hybrid bio microchip to address the effect of psPEF to the intra organelle nanoporation of multilayer osteoblast cell within the microchip. The experimental data are given bellow.

\section{Experimental results}

The below table shows the percentage of permeabilized cells depending both on the number and amplitude of applied electric field. By the influences of above pulse it is observed that at pico pulse a large amount of dedicated chemicals entered into the intra organelle (Table 2).

The effects of pico second electrical pulses on the intra organelle are also demonstrated by the uptake of dyes and the orientation of phosphatidylserine in the membrane after applying pulses. Three of the dyes most often used to study changes in plasma membrane permeability are trypan blue, propidium iodide (PI), and ethidium homodimer. The above figure shows the uptake of PI by osteoblast cells. It is interesting that the increase in fluorescence is observed only after approximately 12 minutes and then completed in one minute. This indicates that the formation of pores large enough to allow passage of propidium iodide is a secondary effect, following the formation of nano pores, which occurs on a pico second timescale. It is likely that the nano pores are too small to allow PI uptake immediately and the secondary PI uptake is due to rapture of intra organelle (Table 3 ).

\section{Characterization of Osteo Nanoporation}

\section{Pore radius}

The Figure 6 shows the variation of intra organelle pore radius along with pole position of applied electric field in different pulse intensity under the influences of pico electric field. It clears that the radius of all the pores are not same it is sinusoidal distributed over the membrane. As the nanopores are created, intra organelle potential increases and the biggest nanopores move just opposite to the equator (E). It is also shown that pore radius is gradually increase as the angle of applied electric field is increase and maximum pore radius is obtain 


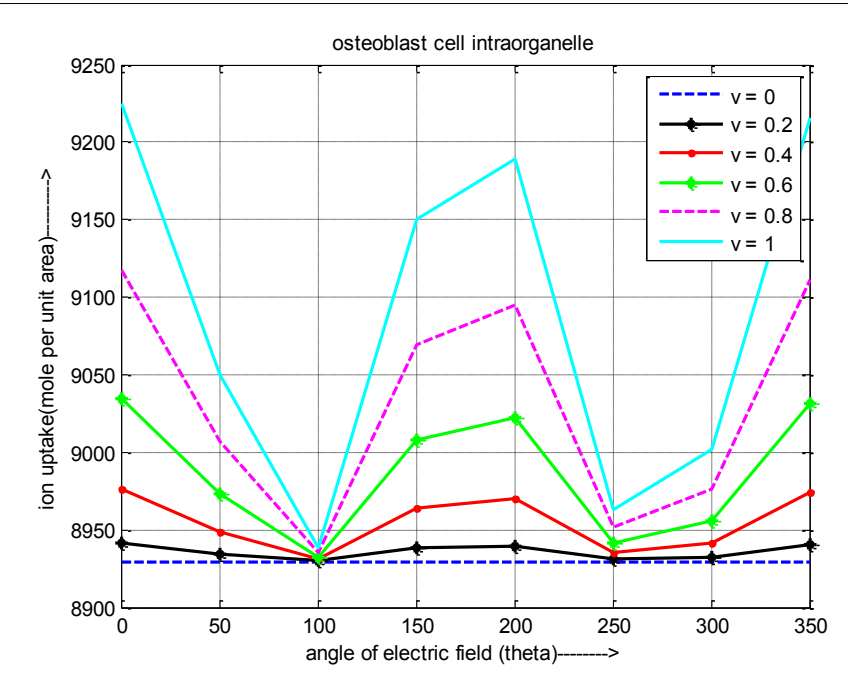

Figure 7: Variation of ion selectivity with respect to azimuthal angle in different pulse intensity.

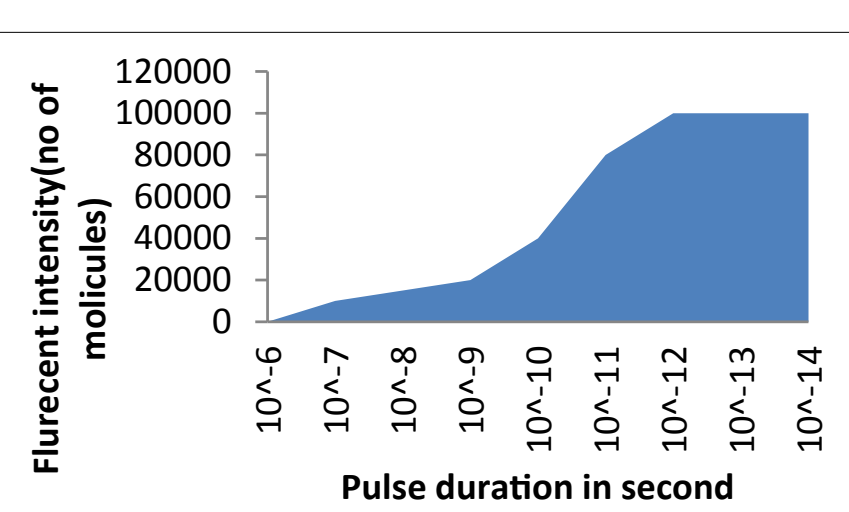

Figure 8: Nanoporation of a typical osteoblast cell undergoing PI uptake.

\section{osteo nanoporation}

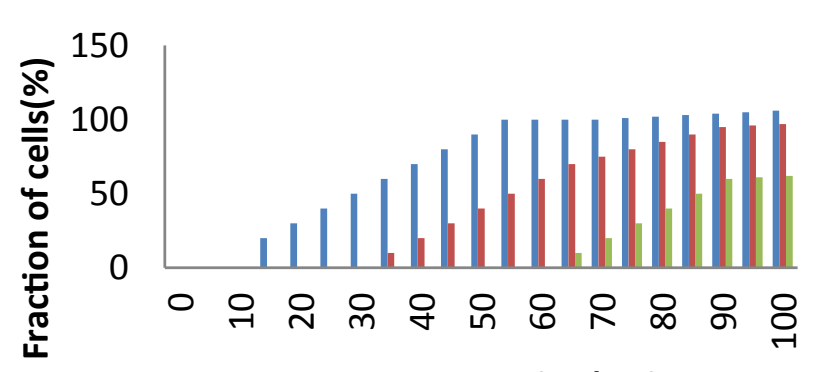

Field strength $(\mathrm{kv} / \mathrm{cm})$

Figure 9: Dependences of the fraction of osteoblast cell, which were nanoporated by a single square-wave electric pulse.

at an angle of $\theta=100$ which is independent of pulse, electrode, micro channel and suspension media specification due to the higher elasticity of layers.

\section{Ion uptake of nanopores}

The Figure 7 shows the variation of intra organelle ion uptake along with pole position of applied electric field for intraorganelle. It is observed that the amount of ion which is uptake by the intra organelle is only occurred at pico scale pulse and its value is not same throughout the whole surface of the layer of the nucleus. The maximum ion uptake occurs at pole $(\theta=100$ and 250$)$ where the surface tension is minimum and it implies that such part of membrane will inherently have an inverse Kelvin vapor pressure effect, that resulting in increased water condensation.

From this observation it is exposed that there is no effect of micro pulse but pico pulse penetrate the on intraorganelle. It supports the numerical and analytical result explore in this research. In this research papers, the fraction of the osteoblast cell that were nanoelectroporated was determined from the experiment as explained above. It should be noted that the right information of the cell nanoporation is rather complex.

Figure 8 shows the dependences of the fraction $(F \mathrm{p})$ of electroporated cells versus the amplitude of the single squarewave electric field pulse, determined for pulses whose duration varied from 50 to $100 \mathrm{ps}$. The amplitude of the electric pulse was changed from 0 to $100 \mathrm{kV} / \mathrm{cm}$. From Figure 9, an increase of the pulse amplitude or duration increases the fraction of the cells being electroporated. For the 100-ps duration pulse, pores permeable to sodium ions appeared in the cell plasma membrane at $10-\mathrm{kV} / \mathrm{cm}$ electric field strength. The field required to electroporate $50 \%$ of the cells in this case was $\sim 20 \mathrm{kV} /$ $\mathrm{cm}$. When the pulse duration was decreased to $75 \mathrm{ps}$, the strength of the electric field required to electroporate $50 \%$ of the cells increased up to $65 \mathrm{kV} / \mathrm{cm}$ In the 50 -ps duration pulse with amplitude of $110 \mathrm{kV} / \mathrm{cm}$, only about $20 \%$ of osteoblast like cells was nanoporated. To date, there has been a lack of studies in which the threshold of the intraorganelle nanoelectroporation was determined for pulses $=100$ ps. However, the fraction of the cells that were electroporated was not estimated. It is observed that when this process for increasing the permeability of the intraorganelle osteoblast like cell membrane (because of picosecond pulse) to the smallest ions $(\mathrm{Na}+, \mathrm{K}+$, and $\mathrm{Rb}+)$ is used, this makes it possible to detect with sufficient accuracy the threshold of the field of poration and allows one to determine the dependence of this field on the pulse duration. It also has to be noted that the electric field strength required to porate the cell plasma membrane using picosecond pulses increases more abruptly with the decrease of the pulse duration.

Since not all cells responded at the same time with a calcium increase (Figures 10 and 11) calcium responses were analyzed in steps of 50 seconds. Thus it was identified the number of cells that reached their peak value in the first 50 seconds, in 100 seconds, etc. It was compared the Peak value in cell number percentage this mean that was compared the percentage of cells that reached their maximum calcium concentration in steps of 50 seconds. It was evident that most cells (50\%) had their maximal calcium increase in the first 50 seconds and

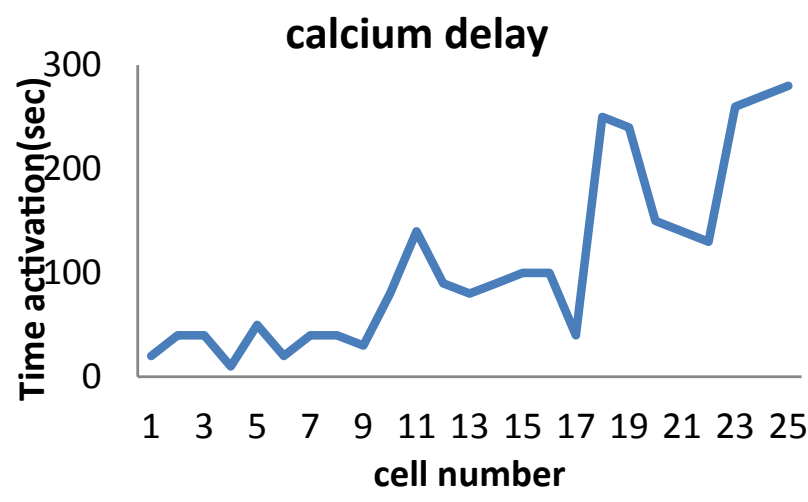

Figure 10: The delay between beginning of stimulation and maximum calcium increase in every cell. 


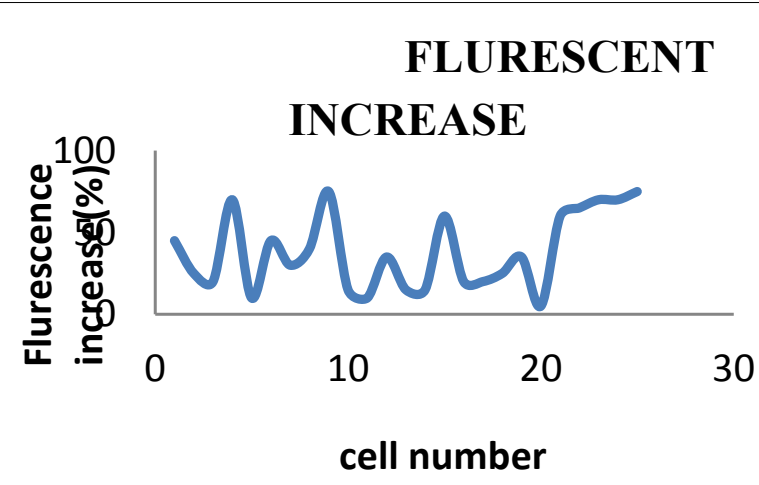

Figure 11: The ratio increase in every cell.

then cell number percentage was decreasing as time went by. Thus a shorter period of stimulation may have a greater influence intracellular rearrangement. This concept is key resources of our idea and we explore concept for nan pore formation over the intraorganelle of osteoblast like cell.

\section{Application}

\section{Drug delivery system}

Practically, intraorganelle nanoporation is used to deliver drugs or other molecules opposed by intraorganelle membranes. Intraorganelle nanoporation has been shown to be safe and effective in humans to overcome with the potential problems for DNA delivery by viruses. It was also observed that only minor histological changes occurred in osteo intraorganelle during nanoporation with either little or no changes in gene profiles nor in DNA stability. One practical medical application for Intraorganelle nanoporation is to permeabilize tumor cell membranes for the delivery of poorly permeable drugs, such as electrochemotherapy and ultra sound in different developed countries.

\section{Tumor cell treatment}

Electro-gene therapy is widely used for cancer treatments. Initial thoughts were to use EGT for replacing defective genes. However, a major focus has been on several basic strategies for cancer therapy, including immune potentiation, suicide gene therapy, restoration of tumor suppressor genes, inhibition of oncogenes, anti-angiogenic gene therapy, delivering genes encoding toxins, or siRNAs to knockdown proteins important for survival and growth. In addition, 2 of 19 patients with non-electroporated distant lesions and no other systemic therapy showed complete regression of all metastases. Cell survival and DNA delivery during intraorganelle nanoporation of course EGT requires that cells survive Intraorganelle nanoporation treatment, so they can express the gene of interest. Finally, there is a threshold that completely compromises cell viability, such that cells are irreversibly permeabilized. It is now clinically used for liver cancer.

\section{In intrinsic and extrinsic cell death}

It was hypothesized that nsPEF-induced apoptosis resulted from supra-intraorganelle nanoporation, this could be due to extrinsic effects on plasma membranes, or intrinsic effects on endoplasmic reticulum, mitochondria and/or nuclei/DNA. lysosomes could also be affecting, but have not been analyzed. When these clones were treated with ten $60 \mathrm{~ns}$ pulses with increasing electric fields $(0-60 \mathrm{kV} / \mathrm{cm})$, there were no differences in electric field effects on cell viability. However, it did show that like other apoptosis stimuli, nsPEFs activate similar can passindependent cell death mechanisms in the absence of caspase activities. To more specifically test for nsPEF-activated extrinsic apoptosis.

\section{Intra cellular cell death activation}

Our present understanding of possible sub cellular targets and cell death mechanisms in response to nsPEFs, and summarizes the finding presented above. Based on the evidence presented above and elsewhere, mitochondria.

\section{Conclusion}

The theoretical model of intra organelle nanoporation of osteoblast cell placed in a 3D hybrid micro biochip under the influences of pico pulse has been reported. This study encourage the specific microchip multiple dimension microfloudic channel with irregular bi metallic ( $\mathrm{Bi}$ and $\mathrm{Au}$ ) side wall electrodes that are designed to deliver a maximum of energy to the biological medium containing multilayer osteoblast cell. The reported nanoporative device aided by a quantitative understanding of the interactions between cells and an external electric field to provide more advantageous conditions than conventional systems with small voltages and power consumptions, continuous flow, small sample volume, and negligible heating.

Intraorganelle nanoporation is a promising technique for targeted drug delivery with various applications in cancer therapy. The initial studies on the treatment of superficial and deep seated tumours with intraorganelle nanoporation showed significant outcomes. To improve the intraorganelle nanoporation as a new modality in cancer treatment, different studies should be performed to determine the mechanisms of action of the technique. Conducting in-vivo and in-vitro experimental, as well as exact modelling studies can shed light on the different aspects of mechanisms of action of this technique. In summary, the present data provide evidence that psPEF introduce the ions with specified microchip possibly through the nucleus mediated pathway. The use of picoseconds pulses not only allows entering a new field of fieldcell interactions, but it may open the door to a range of non-invasive therapeutic applications.

We have designed a micro electroporation device aided by a quantitative understanding of the interactions between cells and an external electric field. The micro device is effective in lying cells while operating at more advantageous conditions than conventional systems: small voltages and power consumptions, continuous flow, small sample volume, and negligible heating. This device has the potential for integration with other micro fluidic components as part of a lab-onchip system for sub cellular analysis, which would greatly facilitate large scale bio medical applications.

\section{Acknowledgement}

We wish to thanks Dr. Soumen Das (IIT, KGP) for assistance with analytical data processing throughout the whole work

\section{References}

1. Marszalek P, Liu DS, Tsong TY (1990) Schwan equation and transmembrane potential induced by alternating electric field. Biophys J 58: 1053-1058.

2. Marty M, Sersa G, Garbay JR, Gehl J,Collins CG, et al. (2006) Electrochemotherapy - An easy, highly effective and safe treatment of cutaneous and subcutaneousmetastases: Results of ESOPE (European Standard Operating Procedures of Electrochemotherapy) study. Ejc Supplements 4: 3-13.

3. Mir LM, Glass LF, Sersa G, Teissie J, Domenge C, et al. (1998) Effective treatment of cutaneous and subcutaneous malignant tumours by electrochemotherapy. British Journal of Cancer 77: 2336-2342.

4. Mir LM (2006) Electrochemotherapy - An easy, highly effective and safe treatment of cutaneous and subcutaneous metastases: Results of ESOPE (European Standard Operating Procedures of Electrochemotherapy) study. Ejc Supplements 4: 3-13.

5. Mir LM, Gehl J, Sersa G, Collins CG, Garbay JR, et al. (2006)Standard operating procedures of the electrochemotherapy: Instructions for the use of bleomycin or cisplatin administered either systemically or locally and electric 
Citation: Sarkar S, Ghose MK (2015) Application and Characterization Osteo Nanoporation under Electrical Stimuli. Gene Technology 4: 123. doi: 10.4172/2329-6682.1000123

Page 7 of 7

pulses delivered by the CliniporatorTM by means of invasive or non-invasive electrodes. Eur J Cancer S4, 14-25.

6. Labanauskiene J, Gehl J, Didziapetriene J (2007) Evaluation of cytotoxic effect of photodynamic therapy in combination with electroporation in vitro. Bioelectrochemistry 70: 78-82.

7. Gothelf A, Gehl J (2010) Gene electrotransfer to skin; review of existing literature and clinical perspectives. Curr Gene Ther 10: 287-299.

8. André F, Mir LM (2004) DNA electrotransfer: its principles and an updated review of its therapeutic applications. Gene Ther 11 Suppl 1: S33-42.

9. Neumann E, Sowers AE, Jordan CA (1989) Electroporation and Electrofusion in Cell Biology, (Eds), in, Plenum, New York

10. Zimmermann U, Neil GA (1996) In: Electromanipulation of Cells, CRC Press, BocaRaton.

11. Pakhomov AG, Miklavcic D, Markov MS (2010) ElectroporationTechniques in Biology in Medicine (Eds.), CRC Press, Boca Raton, p. 528

12. Rubinsky B (2010) Irreversible electroporation (Ed.), Series in Biomedical Engineering,Springer-Verlag, in: Berlin Heidelberg.

13. Craviso GL, Choe S, Chatterjee P, Chatterjee I, Vernier PT (2010) Nanosecond electric pulses: a novel stimulus for triggering $\mathrm{Ca} 2+$ influx into chromaffin cells via voltage-gated Ca2+ channels. Cell Mol Neurobiol 30: 1259-1265.

14. Vernier PT, Sun Y, Gundersen MA (2006) Nanoelectropulse-driven membrane perturbation and small molecule permeabilization. BMC Cell Biol 7: 37

15. Pakhomov AG, Kolb JF, White JA, Joshi RP, Xiao S, et al. (2007) Long-lasting plasma membrane permeabilization in mammalian cells by nanosecond pulsed electric field (nsPEF). Bioelectromagnetics 28: 655-663.

16. Gowrishankar TR, Weaver JC (2006) Electrical behavior and pore accumulation in a multicellular model for conventional and supra-electroporation. Biochem Biophys Res Commun 349: 643-653.

17. Pakhomov AG, Shevin R, White JA, Kolb JF, Pakhomova ON, et al. (2007) Membrane permeabilization and cell damage by ultrashort electric field shocks. Arch Biochem Biophys 465: 109-118.

18. Frey W, White JA, Price RO, Blackmore PF, Joshi RP,et al (2006) Plasma membrane voltage changes during nanosecondpulsed electric field exposure, Biophys. J. 903608-3615.

19. AndreF, MirLM (2004) Gene Ther. 11: S33-S42.

20. André FM, Gehl J, Sersa G, Préat V, Hojman P, et al. (2008) Efficiency of highand low-voltage pulse combinations for gene electrotransfer in muscle, liver tumor, and skin. Hum Gene Ther 19: 1261-1271.

21. Beebe SJ, Schoenbach KH (2005) Nanosecond pulsed electric fields: a new stimulus to activate intracellular signaling. J Biomed Biotechnol 2005: 297-300.

22. Beebe SJ, White J, Blackmore PF, Deng Y, Somers K, et al. (2003) Diverse effects of nanosecond pulsed electric fields on cells and tissues. DNA Cell Biol 22: 785-796.

23. Berenger JP (1996) J Comput Phys 127(2): 363-379.

24. Bowman AM, Nesin OM, Pakhomova ON, Pakhomov AG (2010) Analysis of plasma membrane integrity by fluorescent detection of $\mathrm{TI}(+)$ uptake. $\mathrm{J}$ Membr Biol 236: 15-26.

25. Buescher ES, Smith RR, Schoenbach KH,004. Plasma Sci. IEEE Trans. 32 1563-1572.

26. Dalmay C, CherayM, Pothier A, Lalloué F, Jauberteau MO, et al.(2010) Sens Actuat. A: Phys. ijcaonline.org. 87: 1-8

27. Amari El, Kenaan S, Merla M, Vergne C, Arnaud-Cormos B, et al. (2010) Photon. Technol Lett IEEE 22: 1577-1579.

28. Gothelf A, Gehl J (2010) Gene electrotransfer to skin; review of existing literature and clinical perspectives. Curr Gene Ther 10: 287-299.

29. Ferrier J, Ross SM, Kanehisa J, Aubin JE (1986) Osteoclasts and osteoblasts migrate in opposite directions in response to a constant electrical field.J Cell Physiol 129: 283-288.

30. Brighton CT, Okereke E, Pollack SR, Clark CC (1992) In vitro bone-cel response to a capacitively coupled electrical field. The role of field strength, pulse pattern, and duty cycle. Clin Orthop Relat Res : 255-262.

31. McLeod KJ, Donahue HJ, Levin PE, Fontaine MA, Rubin CT (1993) Electric fields modulate bone cell function in a density-dependent manner. $\mathrm{J}$ Bone Miner Res 8: 977-984. 